Claude-Alain Faure, 23 ch. de Mont-Rose, CH-1294 Genthod, Switzerland François Guénard, Université Paris-Sud, Département de Mathématiques, Bâtiment 425, F-91405 Orsay, France

\title{
UPPER AND LOWER APPROXIMATIONS OF PERRON INTEGRABLE FUNCTIONS
}

\section{Introduction}

Let $f:[a, b] \rightarrow \overline{\mathbb{R}}$ be in a certain class of integrable functions. Then an upper approximation of $f$ is an integrable function $h:[a, b] \rightarrow \overline{\mathbb{R}}$ which satisfies the condition $f \leqslant h$, and some additional properties. For instance, we may look for a function $h_{\varepsilon}$ satisfying a nice regularity property and $\int_{a}^{b}\left(h_{\varepsilon}-f\right)<\varepsilon$, or a less regular function $h$ such that $f(x)=h(x)$ almost everywhere. The two following results are well known for the Riemann and Lebesgue integrals (see Theorem 4.4.3 in [7] and Theorem 15.8 in [6] for Theorem B):

Theorem A For a function $f:[a, b] \rightarrow \mathbb{R}$ the following are equivalent:

1) $f$ is Riemann integrable,

2) for any $\varepsilon>0$ there exist two $R$-integrable functions $g_{\varepsilon}$ and $h_{\varepsilon}$ such that

a) $g_{\varepsilon}$ and $h_{\varepsilon}$ are continuous,

b) $g_{\varepsilon} \leqslant f \leqslant h_{\varepsilon}$ and $\int_{a}^{b}\left(h_{\varepsilon}-g_{\varepsilon}\right)<\varepsilon$,

3) there exist two $R$-integrable functions $g$ and $h$ such that

a) $g \in \mathcal{C}^{+}$and $h \in \mathcal{C}^{-}$(i.e. they are semicontinuous),

b) $g \leqslant f \leqslant h$ and $g(x)=h(x)$ almost everywhere.

Theorem B For a function $f:[a, b] \rightarrow \overline{\mathbb{R}}$ the following are equivalent:

1) $f$ is Lebesgue integrable,

2) for any $\varepsilon>0$ there exist two L-integrable functions $g_{\varepsilon}$ and $h_{\varepsilon}$ such that

Key Words: Perron integration, approximation, semiBaire 1 functions

Mathematical Reviews subject classification: Primary: 26A39, 26A21

Received by the editors July 21, 1995 
a) $g_{\varepsilon} \in \mathcal{C}^{-}$and $h_{\varepsilon} \in \mathcal{C}^{+}, g_{\varepsilon}<\infty$ and $h_{\varepsilon}>-\infty$,

b) $g_{\varepsilon} \leqslant f \leqslant h_{\varepsilon}$ and $\int_{a}^{b}\left(h_{\varepsilon}-g_{\varepsilon}\right)<\varepsilon$,

3) there exist two L-integrable functions $g$ and $h$ such that

a) $g \in \mathcal{C}^{-+}$and $h \in \mathcal{C}^{+-}, g<\infty$ and $h>-\infty$,

b) $g \leqslant f \leqslant h$ and $g(x)=h(x)$ almost everywhere.

Here a function $h \in \mathcal{C}^{+-}$if and only if there exists a decreasing sequence of lower semicontinuous functions $h_{n} \in \mathcal{C}^{+}$which converges to $h$ (cf. Section 3). The main object of this paper is to give a direct proof of the following result.

Theorem C For a function $f:[a, b] \rightarrow \overline{\mathbb{R}}$ the following are equivalent:

1) $f$ is Perron integrable,

2) for any $\varepsilon>0$ there exist two P-integrable functions $g_{\varepsilon}$ and $h_{\varepsilon}$ such that

a) $g_{\varepsilon} \in \mathcal{C}^{+-}$and $h_{\varepsilon} \in \mathcal{C}^{-+}, g_{\varepsilon}<\infty$ and $h_{\varepsilon}>-\infty$,

b) $g_{\varepsilon} \leqslant f \leqslant h_{\varepsilon}$ and $\int_{a}^{b}\left(h_{\varepsilon}-g_{\varepsilon}\right)<\varepsilon$,

3) there exist two P-integrable functions $g$ and $h$ such that

a) $g \in \mathcal{C}^{(+)-+}$and $h \in \mathcal{C}^{(-)+-}, g<\infty$ and $h>-\infty$,

b) $g \leqslant f \leqslant h$ and $g(x)=h(x)$ almost everywhere.

It must be emphasized that the paper is self-contained. No prerequisites are needed, except the basic properties of the Perron integral and some classical results on semicontinuous functions. In particular, we do not use any notion of measure theory (which is alien to the spirit of Perron integration). All we need is an appropriate definition of sets of measure zero.

On the contrary, our Theorem 4.1 provides a new and direct proof of the measurability of Perron integrable functions, and hence of Kurzweil-Henstock integrable functions since both integrals coincide (cf. Theorem 11.5 and Theorem 11.6 in [3], or the original paper of Kurzweil [5]).

Usually, one presents the measurability as a consequence of the following well-known theorem: An integrable function is almost everywhere the derivative of its indefinite integral. But this uses the Vitali Covering Theorem, and therefore the result cannot be introduced at an elementary level. We believe that our demonstration is simpler and more elegant, and that it provides an important short-cut in the exposition of the fashionable theory of KurzweilHenstock integration. So let us sketch the ideas of the proof.

Consider a KH-integrable function $f:[a, b] \rightarrow \mathbb{R}$. Then $f$ is Perron integrable and there exist, for every $n \in \mathbb{N}$, an upper function $M_{n}$ and a lower function $m_{n}$ of $f$ with $M_{n}(b)-m_{n}(b)<1 / n$. We define the functions $g, h:[a, b] \rightarrow$ 
$\overline{\mathbb{R}}$ by $g(x)=\sup \left\{\overline{\mathrm{D}} m_{n}(x) / n \in \mathbb{N}\right\}$ and $h(x)=\inf \left\{\underline{\mathrm{D}} M_{n}(x) / n \in \mathbb{N}\right\}$. Now we remark that the derivates $\overline{\mathrm{D}} m_{n}$ and $\underline{\mathrm{D}} M_{n}$ are measurable (even Baire 2 by a theorem of Hájek, cf. TTheorem 4.2.3 in [1]). Hence the functions $g$ and $h$ are measurable. Using that $M_{n}-m_{n}$ is an upper function of $h-g$ we conclude that $\int_{a}^{b}(h-g)=0$, and we therefore obtain $g(x)=h(x)$ almost everywhere. This proves the measurability of $f$ because we have $g \leqslant f \leqslant h$.

We are very grateful to Arthur Kruse for his careful reading of the paper and many valuable suggestions.

\section{Preliminaries}

Definition 2.1. Let $f:[a, b] \rightarrow \overline{\mathbb{R}}$ be an extended real-valued function. We recall that $M:[a, b] \rightarrow \mathbb{R}$ is an upper function of $f$ if $M(a)=0, \underline{\mathrm{D}} M>-\infty$ and $\underline{\mathrm{D}} M \geqslant f$. The lower functions of $f$ are defined symmetrically.

The function $f:[a, b] \rightarrow \overline{\mathbb{R}}$ is called integrable (in the sense of Perron) if for every $\varepsilon>0$ there exist an upper function $M$ and a lower function $m$ such that $M(b)-m(b)<\varepsilon$.

Lemma 2.2. If $M$ is an upper function of $f$ and $m$ is a lower function of $f$, then the function $M-m$ is increasing.

Proof. We have $\underline{\mathrm{D}}(M-m)(x) \geqslant \underline{\mathrm{D}} M(x)-\overline{\mathrm{D}} m(x) \geqslant 0$ for all $x \in[a, b]$.

In particular, the numbers $\inf \{M(b) / M$ is an upper function of $f\}$ and $\sup \{m(b) / m$ is a lower function of $f\}$ are equal when $f$ is integrable. This common real value is called the integral of $f$, and it is denoted by $\int_{a}^{b} f$.

Definition 2.3. We say that a subset $E \subseteq[a, b]$ is negligible if its characteristic function $\chi_{E}$ is integrable and $\int_{a}^{b} \chi_{E}=0$. As usual, a property is said to hold almost everywhere if the exceptional set is negligible.

Lemma 2.4. Let $f, g:[a, b] \rightarrow \overline{\mathbb{R}}_{+}$be two nonnegative functions with $f \leq g$. If the function $g$ is integrable and $\int_{a}^{b} g=0$, then also $\int_{a}^{b} f=0$.

Proposition 2.5. Let $f_{n}:[a, b] \rightarrow \overline{\mathbb{R}}_{+}$be an increasing sequence of nonnegative functions which converges pointwise to a function $f:[a, b] \rightarrow \overline{\mathbb{R}}_{+}$. If the function $f_{n}$ is integrable and $\int_{a}^{b} f_{n}=0$ for every $n \in \mathbb{N}$, then also $\int_{a}^{b} f=0$.

Proof. For every $n \in \mathbb{N}$ there exists an upper function $M_{n}$ of $f_{n}$ such that $M_{n}(b)<\varepsilon 2^{-n}$. Then the function $M(x):=\sum_{n=1}^{\infty} M_{n}(x)$ is clearly an upper function of $f$ and we have $M(b)<\varepsilon$. 
Corollary 2.6. 1) Any subset of a negligible set is negligible. 2) Any denumerable union of negligible sets is negligible.

The following result has many interesting consequences.

Theorem 2.7. Let $f:[a, b] \rightarrow \overline{\mathbb{R}}_{+}$be any nonnegative function. Then $f$ is integrable and $\int_{a}^{b} f=0$ if and only if $f(x)=0$ almost everywhere.

Proof. $(\Rightarrow)$ It is enough to show that the set $E_{n}:=\left\{x \in[a, b] / f(x) \geqslant \frac{1}{n}\right\}$ is negligible. This follows from Lemma 2.4 because $\chi_{E_{n}} \leqslant n f$ and $\int_{a}^{b} n f=0$.

$(\Leftarrow)$ By hypothesis the set $E:=\{x \in[a, b] / f(x) \neq 0\}$ is negligible. And since $f \wedge n \leqslant n \chi_{E}$, we obtain $\int_{a}^{b} f \wedge n=0$ for every $n \in \mathbb{N}$. So the assertion follows from Proposition 2.5.

Corollary 2.8. Let $H:[a, b] \rightarrow \mathbb{R}$ be an increasing function. Then one has $\underline{\mathrm{D}} H(x)<\infty$ almost everywhere.

Proof. We may assume that $H(a)=0$. We consider the function $f$ defined by $f(x)=\infty$ if $\underline{\mathrm{D}} H(x)=\infty$, and $f(x)=0$ otherwise. Then $\frac{1}{n} H$ is an upper function of $f$ for every $n \in \mathbb{N}$, and this shows that $\int_{a}^{b} f=0$. By the theorem we conclude that $f(x)=0$ almost everywhere.

Corollary 2.9. If $f:[a, b] \rightarrow \overline{\mathbb{R}}$ is an integrable function, then $f(x)$ is finite almost everywhere.

Proof. Let $M$ be an upper function and $m$ a lower function of $f$. We must have $\underline{\mathrm{D}}(M-m)(x)=\infty$ for every $x \in[a, b]$ with $f(x) \notin \mathbb{R}$. So the result is a consequence of the preceding corollary.

Corollary 2.10. Let $E \subseteq[a, b]$ be a negligible subset and $\varepsilon>0$. Then there exists an increasing function $H:[a, b] \rightarrow \mathbb{R}$ with $H(a)=0$ and $H(b)<\varepsilon$, and such that $\underline{\mathrm{D}} H(x)=\infty$ for all $x \in E$.

Proof. Consider the function $f$ defined by $f(x)=\infty$ if $x \in E$, and $f(x)=0$ otherwise. By the theorem we have $\int_{a}^{b} f=0$. So the corollary reduces to the choice of an appropriate upper function.

Corollary 2.11. Let $f:[a, b] \rightarrow \overline{\mathbb{R}}$ be an integrable function. If $g(x)=f(x)$ almost everywhere, then $g$ is integrable and one has $\int_{a}^{b} g=\int_{a}^{b} f$.

Proof. Let $\varepsilon>0$. There exist an upper function $M$ and a lower function $m$ of $f$ such that $M(b)-m(b)<\varepsilon$. Now the set $E:=\{x \in[a, b] / g(x) \neq f(x)\}$ is negligible and we can choose an increasing function $H$ as in the preceding corollary. Then $K:=M+H$ and $k:=m-H$ are upper and lower functions of $g$ respectively and we have $K(b)-k(b)<3 \varepsilon$. 


\section{SemiBaire 1 Functions}

It is well known that a function $f:[a, b] \rightarrow \overline{\mathbb{R}}$ is Baire 1 if and only if the sets $\{x \in[a, b] / f(x)>\alpha\}$ and $\{x \in[a, b] / f(x)<\alpha\}$ are $F_{\sigma}$ for all $\alpha \in \overline{\mathbb{R}}$ (refer to Theorem 11.12 in $[8])$. This motivates the following definition.

Definition 3.1. We say that a function $f:[a, b] \rightarrow \overline{\mathbb{R}}$ is lower semiBaire 1 if the set $\{x \in[a, b] / f(x)>\alpha\}$ is an $F_{\sigma}$ for all $\alpha \in \mathbb{R} \cup\{-\infty\}$. Similarly, we say that the function $f$ is upper semiBaire 1 if the set $\{x \in[a, b] / f(x)<\alpha\}$ is an $F_{\sigma}$ for all $\alpha \in \mathbb{R} \cup\{\infty\}$.

For the proofs of the three following lemmas we just use that the class of $F_{\sigma}$-sets is closed under finite intersections and denumerable unions.

Lemma 3.2. The class of lower semiBaire 1 functions is closed under finite infima and denumerable suprema.

Lemma 3.3. Let $f, g:[a, b] \rightarrow \overline{\mathbb{R}}$ be lower semiBaire 1 . If the sum $f+g$ is well defined, then it is lower semiBaire 1.

Lemma 3.4. Let $f, g:[a, b] \rightarrow \overline{\mathbb{R}}_{+}$be nonnegative and lower semiBaire 1 . If the product $f \cdot g$ is well defined, then it is lower semiBaire 1 .

Proposition 3.5. A function $f:[a, b] \rightarrow \overline{\mathbb{R}}$ is lower semiBaire 1 if and only if there is an increasing sequence of upper semicontinuous functions $f_{n}$, with values in $\mathbb{R} \cup\{-\infty\}$, and converging to $f$ pointwise.

Proof. $(\Rightarrow)$ For every rational $r \in \mathbb{Q}$ the set $\{x \in[a, b] / f(x) \leqslant r\}$ is a $G_{\delta}$. Thus it can be written as an intersection $\bigcap_{n \in \mathbb{N}} U_{r n}$ of open subsets of $[a, b]$. We then define $f_{r n}:[a, b] \rightarrow \mathbb{R} \cup\{-\infty\}$ by

$$
f_{r n}(x)= \begin{cases}-\infty & \text { if } x \in U_{r n} \\ r & \text { otherwise. }\end{cases}
$$

It is easily verified that $f(x)=\sup \left\{f_{r n}(x) / r \in \mathbb{Q}\right.$ and $\left.n \in \mathbb{N}\right\}$ and that each function $f_{r n}$ is upper semicontinuous. Therefore we conclude by considering an enumeration $g_{1}, g_{2}, \ldots$ of these functions and $f_{n}:=g_{1} \vee \cdots \vee g_{n}$.

$(\Leftarrow)$ We use that the sets $\left\{x \in[a, b] / f_{n}(x) \geqslant \alpha+1 / n\right\}$ are closed.

Notation 3.6. According to the proposition we shall denote by $\mathcal{C}^{-+}$the set of all lower semiBaire 1 functions and by $\mathcal{C}^{+-}$the set of all upper semiBaire 1 functions. (As usual, the sets of lower and upper semicontinuous functions are denoted by $\mathcal{C}^{+}$and $\mathcal{C}^{-}$respectively.) 
A bilateral lower derivate $\underline{\mathrm{D}} F$ is not necessarily lower semiBaire 1 . But it is Baire 2 by a theorem of Hájek [4]. And if we examine the proof of Garg, cf. Theorem 3.4 in [2], we remark that the derivate $\underline{D} F$ is lower semiBaire 1 provided the function $F$ satisfies a weak regularity condition: $F(x-) \geqslant F(x)$ and $F(x) \geqslant F(x+)$ whenever the limit exists. In fact, any lower derivate will be lower semiBaire 1 if we consider the following definition.

Definition 3.7. Let $F:[a, b] \rightarrow \mathbb{R}$ and $x \in(a, b)$. Then the two-sided lower derivate of the function $F$ at the point $x$ is the number

$$
\text { D. } F(x):=\sup _{\delta>0} \inf \left\{\frac{F(z)-F(y)}{z-y} / x-\delta<y<x<z<x+\delta\right\} \text {. }
$$

At the endpoints of $[a, b]$ we put $\mathrm{D} \cdot F(a)=\underline{\mathrm{D}} F(a)$ and $\mathrm{D} \cdot F(b)=\underline{\mathrm{D}} F(b)$. The two-sided upper derivate $\mathrm{D}^{\bullet} F(x)$ is defined symmetrically.

Lemma 3.8. One has $\underline{\mathrm{D}} F(x) \leqslant \mathrm{D} . F(x)$ at every point $x \in(a, b)$. Moreover, if the function $F$ is continuous at $x$, then one has $\underline{\mathrm{D}} F(x)=\mathrm{D} \cdot F(x)$.

PROOF. The first assertion follows immediately from the equality

$$
\frac{F(z)-F(y)}{z-y}=\frac{F(z)-F(x)}{z-x} \cdot \frac{z-x}{z-y}+\frac{F(x)-F(y)}{x-y} \cdot \frac{x-y}{z-y}
$$

and the second assertion is left as an exercise.

Theorem 3.9. For any function $F:[a, b] \rightarrow \mathbb{R}$ the two-sided lower derivate D. $F$ is lower semiBaire 1.

Proof. We want to show that the set $E_{\alpha}:=\left\{x \in[a, b] / \mathrm{D}_{\bullet} F(x)>\alpha\right\}$ is an $F_{\sigma}$. For every integer $n \in \mathbb{N}$ we consider the set

$$
E_{\alpha n}:=\left\{x \in[a, b] / y \in I_{n}^{-}(x) \text { and } z \in I_{n}^{+}(x) \text { imply } \frac{F(z)-F(y)}{z-y}>\alpha\right\},
$$

where $I_{n}^{-}(x)=(x-1 / n, x) \cap[a, b]$ and $I_{n}^{+}(x)=(x, x+1 / n) \cap[a, b]$, with the two exceptions $I_{n}^{-}(a)=\{a\}$ and $I_{n}^{+}(b)=\{b\}$. Since $E_{\alpha}$ is trivially the union of the sets $E_{\alpha n}$, it is enough to show that each $E_{\alpha n}$ is closed.

So let $x_{i}$ be a sequence in $E_{\alpha n}$ which converges to a point $x \in(a, b)$, and let $y \in I_{n}^{-}(x)$ and $z \in I_{n}^{+}(x)$. Then $y \in I_{n}^{-}\left(x_{i}\right)$ and $z \in I_{n}^{+}\left(x_{i}\right)$ for every point $x_{i}$ with $\left|x_{i}-x\right|<\min \{y-x+1 / n, x-y, z-x, x-z+1 / n\}$, which proves the required inequality. The cases $x \in\{a, b\}$ are left to the reader.

Remark 3.10. The class of integrable functions would not be affected if we considered two-sided extreme derivatives in the definition of upper and lower functions. This modified Perron integral corresponds to a modified KurzweilHenstock integral, where tags lie in the interior of division intervals. 


\section{The Main Results}

Theorem 4.1. For every integrable function $f:[a, b] \rightarrow \overline{\mathbb{R}}$ there exist $g$ and $h:[a, b] \rightarrow \overline{\mathbb{R}}$ with the following properties:

1) $g \in \mathcal{C}^{+-+}$and $h \in \mathcal{C}^{-+-}$,

2) $g<\infty$ and $h>-\infty$,

3) $g \leqslant f \leqslant h$

4) $g(x)=h(x)$ almost everywhere.

In particular, the functions $g$ and $h$ are integrable (cf. Corollary 2.11).

Proof. By Corollary 2.9 we can choose two functions $f_{1}<\infty$ and $f_{2}>-\infty$ with $f_{1} \leqslant f \leqslant f_{2}$ and $f_{1}(x)=f_{2}(x)$ a.e. We remark that the three integrals are equal, cf. Corollary 2.11. So there exist for every $n \in \mathbb{N}$ a lower function $m_{n}$ of $f_{1}$ and an upper function $M_{n}$ of $f_{2}$ with $M_{n}(b)-m_{n}(b)<1 / n$. Then we consider the functions $g$ and $h$ defined by

$$
g(x)=\sup \left\{\mathrm{D}^{\bullet} m_{n}(x) / n \in \mathbb{N}\right\} \text { and } h(x)=\inf \left\{\mathrm{D} \cdot M_{n}(x) / n \in \mathbb{N}\right\} .
$$

The function $h$ is in $\mathcal{C}^{-+-}$because D. $M_{1} \wedge \cdots \wedge \mathrm{D} . M_{n}$ is lower semiBaire 1 , cf. Theorem 3.9 and Lemma 3.2. In the same way, the function $g$ is in $\mathcal{C}^{+-+}$. Since the properties 2) and 3) are clearly satisfied (according to Lemma 3.8), it remains to verify the last property.

Let $E_{n}$ be the (denumerable) set of discontinuities of $M_{n}-m_{n}$, and let $E$ be the union of the $E_{n}$ 's. We consider the function $k:[a, b] \rightarrow \overline{\mathbb{R}}_{+}$defined by $k(x)=h(x)-g(x)$ if $x \notin E$, and $k(x)=0$ if $x \in E$. Using that

$$
k(x) \leqslant h(x)-g(x) \leqslant \mathrm{D} \cdot M_{n}(x)-\mathrm{D}^{\bullet} m_{n}(x) \leqslant \mathrm{D} \cdot\left(M_{n}-m_{n}\right)(x)
$$

and Lemma 3.8, we deduce that $M_{n}-m_{n}$ is an upper function of $k$ for each integer $n \in \mathbb{N}$. Therefore $k$ is integrable and $\int_{a}^{b} k=0$. From Theorem 2.7 we get $k(x)=0$ almost everywhere, and thus the assertion follows.

The following theorem is a well-known result of the theory of integration. So the details of the proof are left to the reader.

Theorem 4.2. (Monotone Convergence Theorem) Let $f_{n}:[a, b] \rightarrow \overline{\mathbb{R}}$ be an increasing sequence of integrable functions which converges (pointwise) to a function $f:[a, b] \rightarrow \overline{\mathbb{R}}$. If the sequence $\int_{a}^{b} f_{n}$ tends to a limit $\ell \in \mathbb{R}$, then $f$ is integrable and one has $\int_{a}^{b} f=\ell$. 
Proof. By Corollaries 2.9 and 2.11 we may assume that the $f_{n}$ 's are finite. Given $\varepsilon>0$ there exists an integer $n \in \mathbb{N}$ such that $\ell<\int_{a}^{b} f_{n}+\varepsilon$. We take a lower function $m_{n}$ and an upper function $M_{n}$ of $f_{n}$ with $M_{n}(b)-m_{n}(b)<\varepsilon$. For each $i \in \mathbb{N}$ there exists an upper function $N_{i}$ of $g_{i}:=f_{n+i}-f_{n+i-1}$ with $N_{i}(b)<\int_{a}^{b} g_{i}+\varepsilon 2^{-i}$. Then $M(x):=M_{n}(x)+\sum_{i=1}^{\infty} N_{i}(x)$ is an upper function of $f$, and we have $M(b)-m_{n}(b)<3 \varepsilon$. Therefore $f$ is integrable.

Proposition 4.3. Let $f:[a, b] \rightarrow \overline{\mathbb{R}}_{+}$be nonnegative and in the class $\mathcal{C}^{-+-}$. If there exists an increasing function $M:[a, b] \rightarrow \mathbb{R}$ such that $f \leqslant \mathrm{D} . M$, then $f$ is integrable and one has $\int_{a}^{b} f \leqslant M(b)-M(a)$.

Proof. Case 1: $f$ is upper semicontinuous and bounded. Then there exists a decreasing sequence of continuous functions $f_{n}$ which converges to $f$. Since continuous functions are clearly integrable, we deduce that $f$ is integrable by Theorem 4.2. Now let $E$ be the denumerable set of all discontinuities of $M$. We define the function $k:[a, b] \rightarrow \overline{\mathbb{R}}_{+}$by $k(x)=f(x)$ if $x \notin E$, and $k(x)=0$ if $x \in E$. Using that $M(x)-M(a)$ is an upper function of $k$, cf. Lemma 3.8, we get the desired inequality $\int_{a}^{b} f=\int_{a}^{b} k \leqslant M(b)-M(a)$.

Case 2: $f$ is lower semiBaire 1. According to Proposition 3.5 there exists an increasing sequence of bounded upper semicontinuous functions $f_{n}$ which converges to $f$. Clearly, we may assume that each $f_{n}$ is nonnegative. So the assertion follows from Theorem 4.2 and the first case.

Case 3: $f \in \mathcal{C}^{-+-}$. We choose a decreasing sequence of lower semiBaire 1 functions $f_{n}$ which converges to $f$. By Theorem 3.9 and Lemma 3.2 we may assume that each $f_{n}$ satisfies the inequality $f_{n} \leqslant \mathrm{D} . M$. Thus we conclude by applying the Monotone Convergence Theorem once more.

Theorem 4.4. Let $f:[a, b] \rightarrow \overline{\mathbb{R}}$ be an integrable function and let $M$ be an upper function of $f$. Then the two-sided lower derivate D. $M$ is integrable.

ProOF. We consider a function $g \in \mathcal{C}^{+-+}$as in Theorem 4.1, and $m$ a lower function of $g$. Then we have the following inequalities:

$$
0 \leqslant \mathrm{D} \cdot M(x)-g(x) \leqslant \mathrm{D} \cdot M(x)-\mathrm{D}^{\bullet} m(x) \leqslant \mathrm{D} \bullet(M-m)(x) .
$$

The function D. $M-g$ is in $\mathcal{C}^{-+-}$by Theorem 3.9 and Lemma 3.3. Hence it is integrable by the preceding proposition, and this implies that the function D. $M$ is also integrable (by Corollaries 2.9 and 2.11 we may modify D. $M-g$ and $g$ on a negligible set so that the sum is well defined).

Remark 4.5. If $M$ is an upper function of an integrable function $f$, then it can be shown that D. $M(x)=\underline{\mathrm{D}} M(x)$ nearly everywhere. (We use Lemma 3.8 and the elementary fact that $\bar{M}(x)-\int_{a}^{x} f$ is an increasing function.) 


\section{Applications of the Theorems}

Key-Lemma 5.1. Let $g$ and $h:[a, b] \rightarrow \overline{\mathbb{R}}$ be two integrable functions with $g \leqslant h$. If a function $f \in \mathcal{C}^{-+-}$satisfies $g \leqslant f \leqslant h$, then it is also integrable.

Proof. Let $m$ be a lower function of $g$ and $M$ an upper function of $h$. Then we have the following inequalities:

$$
0 \leqslant f(x)-\mathrm{D}^{\bullet} m(x) \leqslant \mathrm{D} \cdot M(x)-\mathrm{D}^{\bullet} m(x) \leqslant \mathrm{D} \cdot(M-m)(x) .
$$

The function $f-\mathrm{D}^{\bullet} \mathrm{m}$ is in $\mathcal{C}^{-+-}$by Theorem 3.9 and Lemma 3.3. Hence it is integrable by Proposition 4.3. Finally, $f=\left(f-\mathrm{D}^{\bullet} m\right)+\mathrm{D}^{\bullet} m$ is integrable by Theorem 4.4. (We also use Corollaries 2.9 and 2.11.)

Corollary 5.2. Let $f, g$ and $h:[a, b] \rightarrow \overline{\mathbb{R}}$ be three integrable functions and suppose that $g \leqslant h$. Then the function $g \vee f \wedge h$ is also integrable.

Proof. Let $f_{1}, g_{1}$ and $h_{1} \in \mathcal{C}^{-+-}$be upper approximations of the functions $f, g, h$ respectively, as in Theorem 4.1. We may assume that $g_{1} \leqslant h_{1}$. Since the function $g_{1} \vee f_{1} \wedge h_{1}$ is in the class $\mathcal{C}^{-+-}$, it is integrable by the previous lemma. So the assertion follows from Corollary 2.11.

Corollary 5.3. Let $f:[a, b] \rightarrow \overline{\mathbb{R}}$ be integrable. Then for any real numbers $\alpha<\beta$ the truncated function $\alpha \vee f \wedge \beta$ is integrable.

Corollary 5.4. Let $f, g$ and $h:[a, b] \rightarrow \overline{\mathbb{R}}$ be three integrable functions and suppose that $f \vee g \leqslant h$. Then the function $f \vee g$ is also integrable.

Corollary 5.5. Let $f$ and $g:[a, b] \rightarrow \overline{\mathbb{R}}$ be two integrable functions. If one has $|f| \leqslant g$, then the function $|f|$ is also integrable.

Proof. The functions $f_{+}=0 \vee f$ and $f_{-}=0 \vee-f$ are integrable.

Corollary 5.6. Let $f$ and $g:[a, b] \rightarrow \overline{\mathbb{R}}$ be two integrable functions with $f$ nonnegative and $g$ bounded. Then the product $f \cdot g$ is also integrable.

Proof. We may assume that $g$ is nonnegative. Let $f_{1}, g_{1} \in \mathcal{C}^{-+-}$be upper approximations of the functions $f, g$ respectively, as in Theorem 4.1 (with $g_{1}$ bounded). By Lemma 3.4 the function $f_{1} \cdot g_{1}$ is in $\mathcal{C}^{-+-}$, and it is integrable by Lemma 5.1. So the assertion follows from Corollary 2.11 .

We conclude this section by showing that the class of integrable functions is closed under left composition with the bounded Borel functions. The proof will use the following well-known result. 
Theorem 5.7. (Dominated Convergence Theorem) Let $f_{n}:[a, b] \rightarrow$ $\overline{\mathbb{R}}$ be a sequence of integrable functions converging to some function $f$. We suppose that there exist two integrable functions $g$ and $h:[a, b] \rightarrow \overline{\mathbb{R}}$ with $g \leqslant f_{n} \leqslant h$ for every $n \in \mathbb{N}$. Then $f$ is integrable, and $\int_{a}^{b} f=\lim _{n \rightarrow \infty} \int_{a}^{b} f_{n}$. Proof. Classical argument using Fatou's Lemma twice.

Proposition 5.8. Let $\varphi: \overline{\mathbb{R}} \rightarrow \mathbb{R}$ be a (bounded) continuous function. Then $\varphi \circ f$ is integrable for every integrable function $f:[a, b] \rightarrow \overline{\mathbb{R}}$.

Proof. By Theorem 4.1 there exists a Baire 3 function $g:[a, b] \rightarrow \overline{\mathbb{R}}$ which satisfies $f(x)=g(x)$ almost everywhere. The function $\varphi \circ g$ is clearly Baire 3 and bounded. By applying the Dominated Convergence Theorem three times we obtain that $\varphi \circ g$ (and hence $\varphi \circ f$ ) is integrable.

Corollary 5.9. Let $\varphi: \overline{\mathbb{R}} \rightarrow \mathbb{R}$ be a bounded Borel function. Then $\varphi \circ f$ is integrable for every integrable function $f:[a, b] \rightarrow \overline{\mathbb{R}}$.

Proof. By transfinite induction using the Dominated Convergence Theorem to show that this property passes through the formation of limits.

\section{Proof of Theorem C}

By the Vitali-Carathéodory Theorem a function $f:[a, b] \rightarrow \overline{\mathbb{R}}$ is measurable if and only if there exist two functions $g \in \mathcal{C}^{-+}$and $h \in \mathcal{C}^{+-}$with $g \leqslant f \leqslant h$ and $g(x)=h(x)$ almost everywhere. (For the case of a bounded $f$ this follows from Theorem B.) Here we shall use the following particular case:

Proposition 6.1. Let $f:[a, b] \rightarrow \overline{\mathbb{R}}$ be lower semiBaire 1 . Then there exists an upper semiBaire 1 function $h:[a, b] \rightarrow \overline{\mathbb{R}}$ with $f \leqslant h$ and $f(x)=h(x)$ a.e.

Proof. We first suppose that $f$ is bounded. By Proposition 3.5 there exists an increasing sequence of bounded upper semicontinuous functions $f_{n}$ which converges to $f$. For every $n \in \mathbb{N}$ there exists a decreasing sequence of continuous functions $f_{n k}$ which converges to $f_{n}$. We may assume that the $f_{n k}$ 's are globally bounded. And by the Monotone Convergence Theorem we may also assume that $\int_{a}^{b}\left(f_{n k}-f_{n}\right)<1 / 2^{n+k}$. Now we put $h_{n k}:=f_{1 k} \vee \cdots \vee f_{n k}$. For each $k \in \mathbb{N}$ the sequence $h_{n k}$ is increasing, and hence it converges to a lower semicontinuous function $h_{k} \geqslant f$. Then $h_{n k}-f \leqslant \sum_{i=1}^{n}\left(f_{i k}-f_{i}\right)$ implies

$$
\int_{a}^{b}\left(h_{n k}-f\right) \leqslant \sum_{i=1}^{n} \int_{a}^{b}\left(f_{i k}-f_{i}\right) \leqslant \sum_{i=1}^{n} 1 / 2^{i+k} \leqslant 1 / 2^{k},
$$

and we thus get $\int_{a}^{b}\left(h_{k}-f\right) \leqslant 1 / 2^{k}$ by the Monotone Convergence Theorem. Since the sequence $h_{k}$ is decreasing, it converges to a function $h \in \mathcal{C}^{+-}$with 
$h \geqslant f$. We remark that $\int_{a}^{b}(h-f)=0$. Finally, by Theorem 2.7 we conclude that $f(x)=h(x)$ almost everywhere. The case of an unbounded function $f$ is proved by considering the function arctan $\circ f$.

Corollary 6.2. Let $f:[a, b] \rightarrow \overline{\mathbb{R}}$ be in the class $\mathcal{C}^{-+-}$. Then there exists an upper semiBaire 1 function $h:[a, b] \rightarrow \overline{\mathbb{R}}$ with $f \leqslant h$ and $f(x)=h(x)$ a.e.

Proof. There exists a decreasing sequence of functions $f_{n} \in \mathcal{C}^{-+}$which converges to $f$. For each $n \in \mathbb{N}$ there exists an upper semiBaire 1 function $h_{n}$ as in the previous proposition. Then the function $h(x):=\inf \left\{h_{n}(x) / n \in \mathbb{N}\right\}$ is also upper semiBaire 1 (cf. Lemma 3.2), and the assertion is proved.

Theorem 6.3. (C) For a function $f:[a, b] \rightarrow \overline{\mathbb{R}}$ the following are equivalent:

1) $f$ is integrable,

2) for any $\varepsilon>0$ there exist two integrable functions $g_{\varepsilon}$ and $h_{\varepsilon}$ such that

a) $g_{\varepsilon} \in \mathcal{C}^{+-}$and $h_{\varepsilon} \in \mathcal{C}^{-+}, g_{\varepsilon}<\infty$ and $h_{\varepsilon}>-\infty$,

b) $g_{\varepsilon} \leqslant f \leqslant h_{\varepsilon}$ and $\int_{a}^{b}\left(h_{\varepsilon}-g_{\varepsilon}\right)<\varepsilon$,

3) there exist two integrable functions $g$ and $h$ such that

a) $g \in \mathcal{C}^{-+}$and $h \in \mathcal{C}^{+-}, g<\infty$ and $h>-\infty$,

b) $g \leqslant f \leqslant h$ and $g(x)=h(x)$ almost everywhere.

Proof. $(1 \Rightarrow 2)$ Consider a lower function $m$ and an upper function $M$ of $f$ with $M(b)-m(b)<\varepsilon$. We put $g_{\varepsilon}=\mathrm{D}^{\bullet} m$ and $h_{\varepsilon}=\mathrm{D} . M$. We have $g_{\varepsilon} \in \mathcal{C}^{+-}$ and $h_{\varepsilon} \in \mathcal{C}^{-+}$by Theorem 3.9. The functions are integrable by Theorem 4.4. For the inequality $\int_{a}^{b}\left(\mathrm{D}_{\cdot} M-\mathrm{D}^{\bullet} m\right)<\varepsilon$ we use Proposition 4.3 together with the formula D. $M(x)-\mathrm{D}^{\bullet} m(x) \leqslant \mathrm{D} \cdot(M-m)(x)$.

$(1 \Rightarrow 3)$ This follows from Theorem 4.1 and the preceding corollary.

Problem 1 Characterize the integrable functions $f:[a, b] \rightarrow \overline{\mathbb{R}}$ that can be approximated by two Baire 1 functions $g_{\varepsilon}$ and $h_{\varepsilon}$.

Problem 2 Find a direct proof of the differentiability a.e. of the indefinite integral (which does not use the Vitali Covering Theorem).

\section{References}

[1] A. Bruckner, Differentiation of Real Functions, Lecture Notes Math., 659, Springer, Berlin, 1978.

[2] K. M. Garg, On bilateral derivates and the derivative, Trans. Amer. Math. Soc., 210 (1975), 295-329. 
[3] R. A. Gordon, The Integrals of Lebesgue, Denjoy, Perron and Henstock, American Mathematical Society, Providence, 1994.

[4] O. Hájek, Note sur la mesurabilité B de la dérivée supérieure, Fund. Math., 44 (1957), 238-240.

[5] J. Kurzweil, Generalized ordinary differential equations and continuous dependence on a parameter, Czechoslovak Math. J., 7 (1957), 418-446.

[6] E. J. McShane, Integration, Princeton University Press, Princeton, 1947.

[7] W. F. Pfeffer, The Riemann Approach to Integration, Cambridge Univ. Press, Cambridge, 1993.

[8] A. C. M. van Rooij, W. H. Schikhof, A Second Course on Real Functions, Cambridge University Press, Cambridge, 1982.

[9] S. Saks, Theory of the Integral, Hafner, Warszaw, 1937. 